Int. J. Dev. Biol. 60: 21-28 (2016)

doi: $10.1387 / \mathrm{ijdb} .160040 \mathrm{mk}$

\title{
Bone morphogenetic protein 4 promotes craniofacial neural crest induction from human pluripotent stem cells
}

\author{
SUMIYO MIMURA ${ }^{\#, 1,2}$, MIKA SUGA $^{\#, 1}$, KAORI OKADA ${ }^{1}$, MASAKI KINEHARA $^{1,3}$, HIROKI NIKAWA $^{2}$ and \\ MIHO K. FURUE*,1

\begin{abstract}
${ }^{1}$ Laboratory of Stem Cell Cultures, National Institutes of Biomedical Innovation, Health and Nutrition, Ibaraki, Osaka, Japan, ${ }^{2}$ Department of Oral Biology \& Engineering Integrated Health Sciences, Institute of Biomedical and Health Sciences, Hiroshima University, Hiroshima, Japan and ${ }^{3}$ Department of Cellular and Molecular Biology, Basic Life Sciences, Institute of Biomedical \& Health Sciences, Hiroshima University, Hiroshima, Japan
\end{abstract}

\begin{abstract}
Neural crest (NC) cells are a group of cells located in the neural folds at the boundary between the neural and epidermal ectoderm. Cranial NC cells migrate to the branchial arches and give rise to the majority of the craniofacial region, whereas trunk and tail NC cells contribute to the heart, enteric ganglia of the gut, melanocytes, sympathetic ganglia, and adrenal chromaffin cells. Positional information is indispensable for the regulation of cranial or trunk and tail NC cells. However, the mechanisms underlying the regulation of positional information during human NC induction have yet to be fully elucidated. In the present study, supplementation of bone morphogenetic protein (BMP) 4 in defined serum-free culture conditions including fibroblast growth factor-2 and Wnt3a from day 8 after NC specification induced the expression of cranial NC markers, AP2 $\alpha$, $M S X 1$, and $D L X 1$, during NC cell differentiation from human pluripotent stem cells. On the other hand, the proportion of cells expressing p75 ${ }^{\text {NTR }}$ or HNK1 decreased compared with that of cells cultured without BMP4, whereas gene expression analysis demonstrated that the expression levels of cranial NC-associated genes increased in BMP4-treated NC cells. These BMP4-treated NC cells were capable of differentiation into osteocytes and chondrocytes. The results of the present study indicate that BMP4 regulates cranial positioning during NC development.
\end{abstract}

KEY WORDS: cranial neural crest, BMP signaling, homeobox gene, human embryonic stem cell, defined culture condition

\section{Introduction}

Neural crest (NC) cells are a group of cells located in the neural folds at the boundary between the neural and epidermal ectoderm. In vertebrates, they delaminate from the dorsal ridges of the neural tube and then migrate extensively within the developing embryo during the process of neurulation (Morikawa et al., 2009). NC cells in the cranial region migrate to the branchial arches and give rise to the majority of cranial mesenchyme that eventually differentiates into odontoblasts, cartilage, craniofacial bone, and connective tissue; a subset of these cells differentiate into cranial ganglia. Cardiac NC cells contribute to heart development and vagal NC cells contribute to the development of enteric ganglia of the gut. Trunk NC cells migrate along the dorsal and lateral pathway and give rise to melanocytes, sympathetic ganglia, and adrenal chromaffin cells (Santagati and Rijli, 2003).

Several studies have demonstrated that neural and NC induction in various species, such as fish and amphibians, is regulated by several signaling pathways, such as TGF $\beta$ signaling, including Activin/Nodal, fibroblast growth factor (FGF) signaling, Wnt signaling, and bone morphogenetic protein (BMP) signaling (Baker etal., 1999, Furue and Asashima, 2004, Patthey et al., 2009). In mice, we previ-

\footnotetext{
Abbreviations used in this paper: BMP, bone morphogenetic protein; BSA, bovine serum albumin; ECM, extra cellular matrix; ESCs, embryonic stem cells; FGF, fibroblast growth factor; HOX gene, Homeobox gene; hESGs, human embryonic stem cells; NC, neural crest; NCSCs, neural crest stem cells; qRT-PCR, quantitative real-time reverse transcription polymerase chain reaction.
}

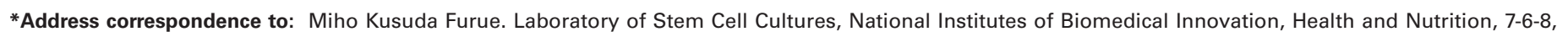
Saito-Asagi, Ibaraki, Osaka 567-0085, Japan. Tel: 81-72-641-9819. Fax: 81-72-641-9812. E-mail: mkfurue@nibiohn.go.jp

\# These authors equally contributed to the study.

Supplementary Material (one figure and three tables) for this paper is available at: http://dx.doi.org/10.1387/ijdb.160040mk

Accepted: 21 January 2016; Edited by: Makoto Asashima

ISSN: Online 1696-3547, Print 0214-6282

(c) 2016 UPV/EHU Press

Printed in Spain 
ously reported that culture in the presence of BMP4 in combination with FGF-2 induces the up-regulation of NC marker genes, including AP2 $\alpha$ and PO protein, during the differentiation of mouse embryonic stem cells (ESCs) into NC cells under chemically defined conditions (Aihara et al., 2010, Furue, 2012). Because culture systems for the stable expansion of human pluripotent stem cells had been developed (Amit et al., 2000, Furue et al., 2008, Kinehara et al., 2013), in vitro studies producing NC cells from human pluripotent stem cells have been reported (Fukuta et al., 2014, Lee et al., 2010, Menendez et al., 2013, Menendez et al., 2011). Dalton et al. (Menendez et al., 2013, Menendez et al., 2011) have demonstrated that a combination of Wnt activation and Smad inhibition under serum-free culture conditions directs the differentiation of human ESCs (hESCs) and induced pluripotent stem cells into NC stem cells (NCSCs).

BMP signaling is well known to inhibit early neural induction. Therefore, the inhibition of BMP signaling, particularly by extracellular antagonists, such as Noggin and Chordin, is usually used for neural induction (Piccolo et al., 1996, Zimmerman et al., 1996). However, Dalton et al. (Menendez et al., 2011) demonstrated that active suppression of BMP signaling by the addition of Noggin is not required for NCSC induction because of the low level of basal BMP-dependent Smad 1, 5, and 8 activity in undifferentiated and differentiating ESCs. On the other hand, we previously demonstrated that AP $2 \alpha$, a premigratory and migratory NC cell marker (Gajavelli et al., 2004), was up-regulated in cells cultured in the presence of recombinant BMP4 for 10 days following culture with recombinant FGF-2 for 4 days in a study using mouse ESCs (Aihara et al., 2010). Sasai et al. (Kamiya et al., 2011) reported that late exposure to BMP4 induces neural ectodermal differentiation of mouse ESCs. However, the directed induction of cranial NC cells, which contribute to the development of craniofacial bone and connective tissue, has yet demonstrated. Whereas positional information is considered to be indispensable for $\mathrm{NC}$ development in vivo, its importance during human NC induction in vitro is currently unknown.

The present study describes the effect of BMP4 on NC induction in vitro and the development of an efficient protocol for the induction of cranial NC cells from hESCs, building on the achievements of previous studies (Aihara et al., 2010, Furue, 2012, Menendez et al., 2013, Menendez et al., 2011). Cranial NC cells were induced by late exposure to recombinant BMP4 in combination with recombinant FGF-2 followed by NC specification. Gene expression induced in the cells by our protocol was comprehensively analyzed, which indicated high expression of migratory cranial NC/pharyngeal arch mesenchyme markers in induced cells. We further confirmed the osteogenic and chondrogenic potential of induced cranial NC cells. The results of the present study demonstrate the specific induction of cranial NC in vitro by BMP4 treatment in a finely timed manner.

\section{Results}

\section{MSX and DLX gene expression in response to BMP4 treatment during NC induction}

To examine the effect of BMP4 on the induction of NC from hESCs, we tested the NCSC induction method previously reported by Dalton et al. (Menendez et al., 2013, Menendez et al., 2011). NCSC induction was driven by treatment with recombinant FGF-2, TGF- $\beta$ /Activin inhibitor SB431542, and recombinant Wnt-3a. We previously reported that late exposure to BMP4 induces the neural ectodermal differentiation of mouse ESCs. From this finding, we hypothesized that the initiation timing of BMP4 treatment affects positional information. To monitor the effects of BMP4 during NC cell differentiation from hESCs, treatment with recombinant BMP4 was initiated at various time points (Fig.1 A) and the expression of $A P 2 \alpha$ was assessed on day 12 by quantitative real-time reverse transcription polymerase chain reaction (qRT-PCR) (Fig.1 B). Expression levels of $A P 2 \alpha$ were higher in the cells treated with BMP4 compared with those treated without BMP4 on day 12.

We also examined the effect of BMP4 on the expression of the positional information genes, $M S X 1$ and $D L X 1$, in cells cultured under the conditions described above. The expression of MSX1 and $M X S 2$ genes, in addition to $A P 2 \alpha$, has been reported in both premigratory and migratory craniofacial NC cells and the craniofacial/ pharyngeal arch mesenchyme (Ishii et al., 2005). DLX genes are expressed in the developing cranial NC cell derivatives, particularly

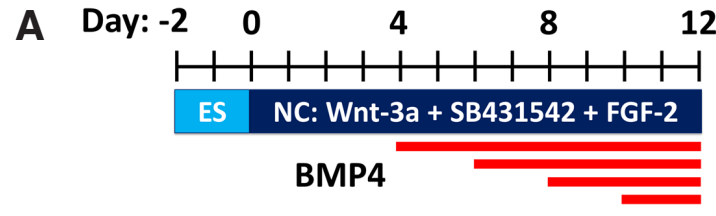

B

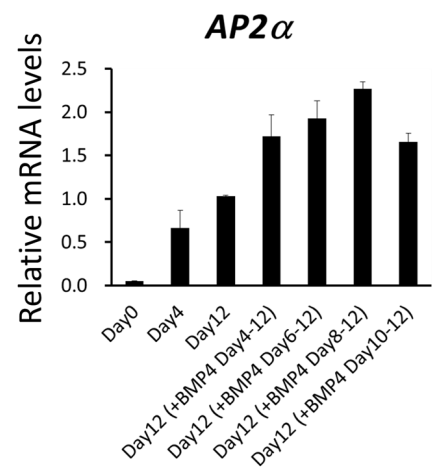

MSX1

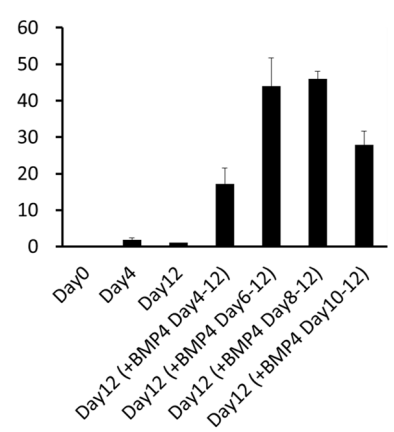

$D L X 1$

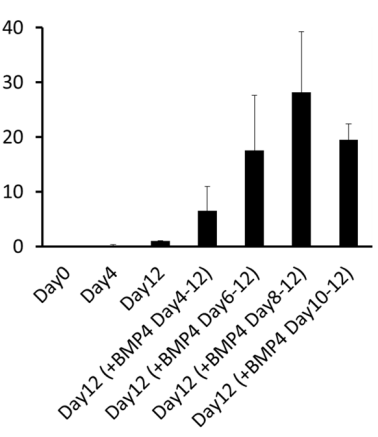

Fig. 1. Altered neural crest (NC) gene expression in response to BMP4 treatment during NC induction. (A) BMP4 was added to cultures at the indicated time points during NC differentiation. ES, hESF9ESC culture medium; NC, NC differentiation medium. (B) Relative mRNA expression levels of AP2 $\alpha, \mathrm{MSX} 1$, and DLX1 on culture days 0,4 , and 12 were analyzed using qRT-PCR. Expression levels were normalized against the housekeeping gene GAPDH. Samples indicated by day 0 , day 4, and day 12 were cultured without BMP4. Day 12 samples were cultured with BMP4 during the periods shown in (A). The relative expression level of each gene is shown with values of cells cultured in NC (without BMP4) on day 12 defined as 1.0. Values are presented as mean $\pm S D(n=3)$. 
in the first and second pharyngeal arches, but not in premigratory or migratory NC cells (Park et al., 2004). In the absence of BMP4, although $A P 2 \alpha$ expression increased during culture, no changes in the expression of $M S X 1$ or $D L X 1$ were observed during the 12-day culture (Fig.1 B). On the other hand, on days 4-12, treatment with BMP4 considerably increased the expression of $M S X 1$ or $D L X 1$, and on days $6-12$, that of $M S X 1, D L X 1$, and $A P 2 \alpha$ considerably increased. Furthermore, on days 8-12, DLX1 and $A P 2 \alpha$ expression greatly increased compared with the other time points. The effect of BMP4 treatment on days 10-12 was comparable with those on days 4-12. Treatment with BMP4 on days 8-12 induced higher gene expression of $A P 2 \alpha, M S X 1$, and $D L X 1$ than that during other days. These results indicate that BMP4 treatment on days 8-12 in combination with FGF-2 and Wnt-3a induces the generation of NC cells with cranial positional information from hESCs. We designated this culture condition as NC-B culture condition.

\section{Characterization of cells induced by activation of BMP4 signaling during NC cell differentiation}

To verify the induction of cranial NC cells from hESCs by NC-B culture condition described above, we next examined the expression profiles of NC markers in induced cells compared with those of cells cultured in the NC culture condition (Fig.2A). First, the expression of the classical NCSC markers, p75 ${ }^{\mathrm{NTR}}$ (Gajavelli etal., 2004) and HNK1 (Nagase et al., 2003), by induced cells was examined by immunocytochemistry (Fig.2 B). The NC culture condition promoted robust specification of $\mathrm{p} 75^{\mathrm{NTR}}$ and HNK1-expressing NCSCs, as described by Menendez et al. (Menendez et al., 2011). In contrast, major popu-
A

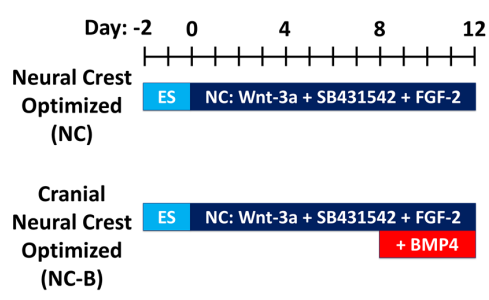

C

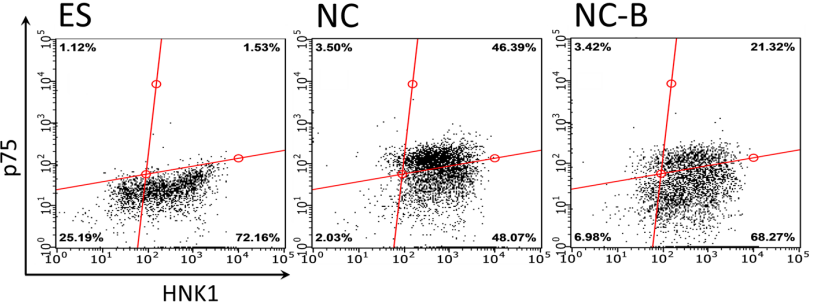

B

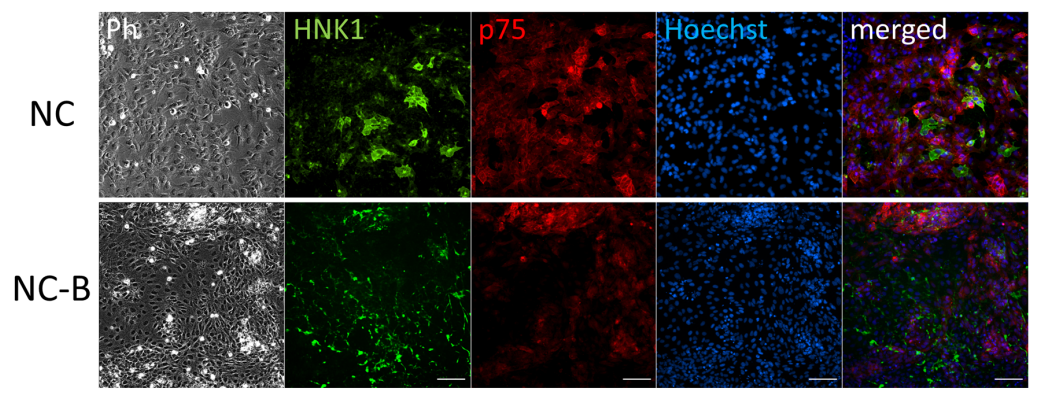

D
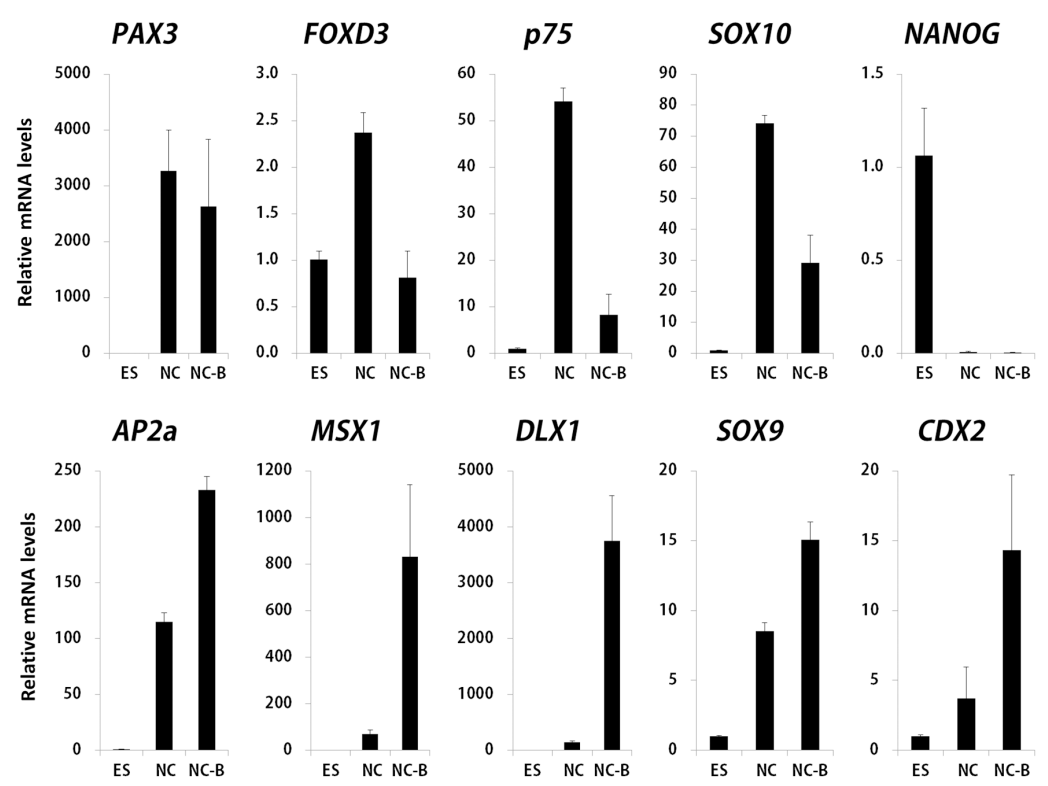

OCT4

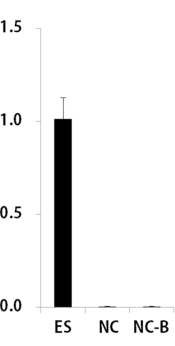

PAX6

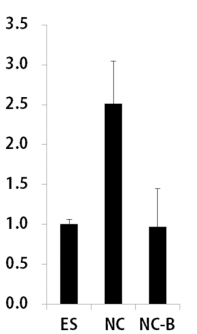

Fig. 2. Characterization of cells induced by the modified neural crest differentiation protocol. (A) Scheme of the neural crest differentiation and the modified cranial neural crest differentiation (NC-B) protocols. (B) H9 human embryonic stem cells were cultured according to the NC or NC-B protocols. Cells were then fixed on day 12 and stained with antibodies for HNK1 and p75. Nuclei were detected by staining with Hoechst33342. Scale bar, $100 \mathrm{~mm}$. (C) Flow cytometry analysis of undifferentiated H9 (ES, left) and neural crest cells derived from $\mathrm{H} 9$ cells cultured in NC (middle) and NC-B (right) culture conditions. Flow cytometry was performed using HNK1/p75 antibodies. The percentage of cells in each quadrant is indicated. (D) Relative gene expression levels in NC cells derived from H9 cells cultured in NC and NC-B culture conditions. Expression levels were normalized against the housekeeping gene GAPDH. The relative expression level of each gene is shown with values of cells cultured in NC (without BMP4) on day 12 defined as 1.0. Values are presented as mean \pm $S D(n=3)$ 

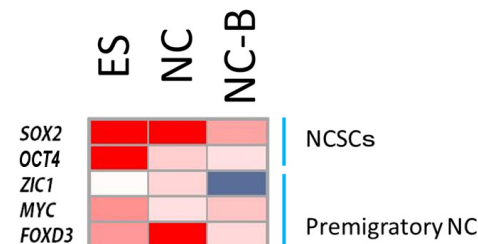

PAX7

PAX7

PAX3
SOX10

PAX6

EMX1

BARX1

SOX9

TWIST

SNAI2

ALX1

ALX4

PITX1

PITX2

MITX2
MSX1
MSX2

DLX1
DLX2

DLX3

DLX4

DLX5

DLX6

TLX1

HOXA1
HOXA2

HOXB1

HOXB2

HOXB3

HOXD1

HOXD3

HAND2

RUNX2

GATA4

GATA6

RUNX1

POU4F1

LHX1

ASCL1

MYCN

PHOX2

LBX2

SOX8

HLX

HOXA5

HOXB4

HOXB5

HOXD4

HOXA7

HOXA9
HOXB7

HOXB8

HOXB9

HOXC6

HOXC8

HOXC9

HOXD8

HOXC10

HOXC11

HOXC12

HOXC13

HOXD11

HOXD12

HOXD13

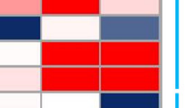

Pratory NC

Ocular mecenchyme

Non-melanocyte NC

First and second pharyngeal arches

Early NC, pharyngeal arches

Premigratory NC, migratory NCCs

Craniofacial cartilage, craniofacial skeleton

Migratory cranial NCCs, teeth, mandible

Cranial NCCs, pharyngeal arches

DRGs, PNS, Sensory neurons

Sympathoadrenal lineage

Cardiac NCCs

Vagal NCCs, ENS

Sacral NCCs

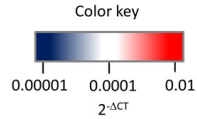

Fig. 3. Microarray analysis demonstrating the up-regulation of cranial neural crest genes in cells cultured in neural crest differentiation medium containing BMP4 (NC-B). Heat map of the expression profiles of 72 genes in undifferentiated H9 cells (ES, $n=3$ ), and NC cells derived from $\mathrm{H} 9$ cells cultured in NC $(n=3)$ and NC-B $(n=6)$ conditions (Fig. 2A). Average delta CT values are shown in Table S3. Blue, red, and green lines indicate early NC, cranial NC, and migratory and posterior neural crest marker genes, respectively. lations of cells induced by NC-B culture condition expressed $\mathrm{p} 75^{\mathrm{NTR}}$ or HNK1 at low levels and displayed different morphology compared with that of $\mathrm{p} 75^{\mathrm{NTR}+} / \mathrm{HNK} 1^{+}$cells cultured in the NC culture condition. To quantify the population of $\mathrm{p} 75^{\mathrm{NTR}+} / \mathrm{HNK} 1{ }^{+}$cells, flow cytometry of cells cultured in each condition was performed (Fig.2 C). The flow cytometric profile of $\mathrm{p} 75^{\mathrm{NTR}}$ and HNK1 expression in day 12 cells induced by NC-B culture condition was compared with that by NC culture condition, indicating that NC-B culture condition decreased the yield of $\mathrm{p} 75^{\mathrm{NTR}+} / \mathrm{HNK} 1^{+}$cells, whereas the HNK1 single-positive $\left(p 75^{\mathrm{NTR}-} / \mathrm{HNK} 1^{+}\right)$cell population was increased.

p75 ${ }^{\text {NTR }}$ and HNK1 have been used to enrich human ES-derived multipotent NCSC cells because these cell surface markers are convenient and useful for staining and sorting living cells (Lee et al., 2010, Menendez et al., 2013, Menendez et al., 2011). However, a previous study reported that both $\mathrm{p} 75^{\mathrm{NTR}-}$ and $\mathrm{p} 75^{\mathrm{NTR}+}$ cells express other NC markers and are able to differentiate into NC cell derivatives in vitro (Curchoe et al., 2010). A further study of migratory NC cells in early human embryos demonstrated that in vivo $\mathrm{p} 75^{\mathrm{NTR}+} / \mathrm{HNK} 1^{+}$ cells were localized to a small subset of migratory NC cells and cells of the ventral neural tube in vivo (Betters et al., 2010). It is likely that additional markers are required for definitive characterization of NC cell subsets derived hESC cultures. Therefore, we further examined the expression levels of the following genes by qRT-PCR analysis (Fig.2 D): well-established NC markers, PAX3, FOXD3, p75 NTR, $S O X 10, S O X 9, A P 2 \alpha, M S X 1$, and $D L X 1$; the neuroectoderm/forebrain and ocular marker, $P A X 6$; the hindbrain/posterior neural tube marker, $C D X 2$; and markers of undifferentiated ES cell state, NANOG and OCT4. NANOG and OCT4 expression levels were significantly decreased in cells induced by both $\mathrm{NC}$ and NC-B culture conditions. It was also confirmed that $P A X 3, F O X D 3, p 75^{N T R}$, and SOX10 were prominently expressed in cells induced by NC condition, corroborating the study by Menendez et al. demonstrating the abundance of premigratory NCSCs (Menendez et al., 2011). On the other hand, in the cells induced by NC-B culture condition, PAX3 expression was maintained at a high level, whereas SOX10 expression was lower than that in $\mathrm{NC}$ condition, and FOXD3 and PAX6 expression levels were the same as those observed in undifferentiated ES cells. In agreement with the above immunostaining results, cells induced by NC-B culture condition expressed significantly lower levels of p75 NTR mRNA than those by NC culture condition. Cells induced by NC-B culture condition expressed high levels of $S O X 9, A P 2 \alpha$, and $C D X 2$. Moreover, we observed robust expression of the cranial NC markers MSX1 and $D L X 1$, specifically in cells induced by NC-B culture condition (Figs.1 B \& 2 D). These results demonstrate that our NC-B culture condition induced a different subset of NC cells, which expressed cranial NC markers, compared with cells induced by $\mathrm{NC}$ culture condition.

\section{Analysis of anterior-posterior identities of the NC cells induced by BMP4 activation}

Furthermore, gene expression was evaluated in hESC derived NC cells to define anterior-posterior positional identities using a customized qRT-PCR array set of 156 genes including $76 \mathrm{NC}$-related genes and 80 HOXgenes (Table S2, S3, Fig.S3). The 72 NC-related and $H O X$ genes were transcription factors with defined expression profiles and functions in NC cells (Curchoe et al., 2010, Nelms and Labosky, 2010). Comparison of expression profiles of the 72 genes in NC cells induced by NC or NC-B culture condition demonstrated that both conditions induced the expression of $A P 2 \alpha, M S X 1$, and 

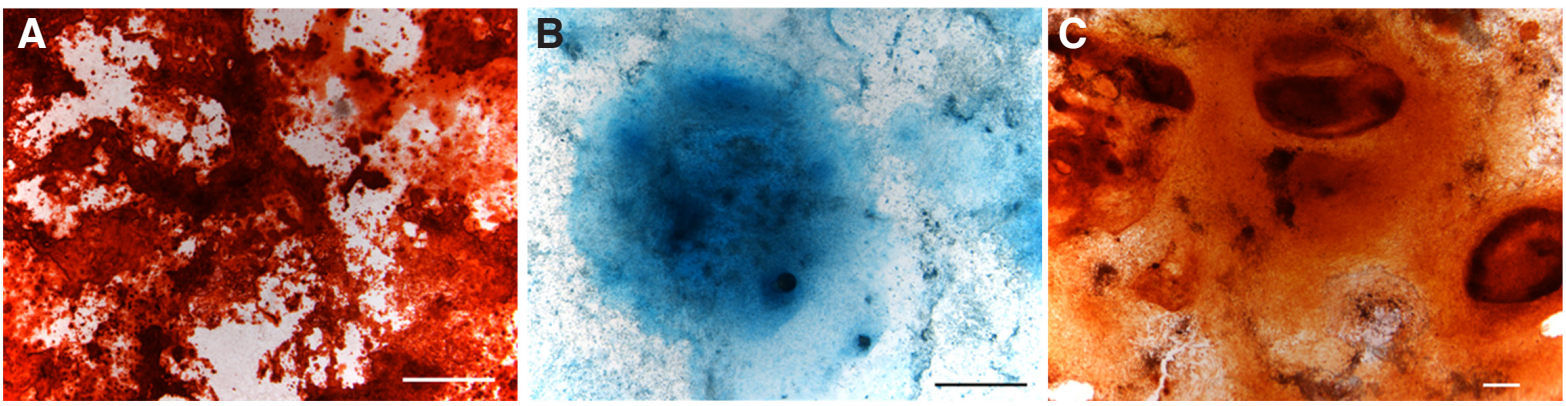

Fig. 4. Differentiation of neural crest cells cultured in BMP4-containing medium. (A) Osteocytes analyzed by alizarin red staining. (B) Chondrocytes analyzed by alcian blue staining. (C) Chondrocytes analyzed by safranine O staining. Scale bars, $1 \mathrm{~mm}$.

MSX2 genes, which are abundant in both early NC and mesenchymal NC cells in pharyngeal arches (Betters et al., 2010, Brunskill et al., 2014, Nelms and Labosky, 2010) (Fig.3, Table S3). Cells induced by the NC culture condition had high expression of the early NC markers, FOXD3 and PAX3, in addition to PAX7, PAX8, SOX10, and SNAI2 (Fig.3, Table S3). Moreover, the expression of posterior $\mathrm{HOX}$ genes was not observed in cells induced by the NC culture condition; however, the expression of anterior HOX genes, OTX1 and OTX2, was observed (Table S3). Both of SOX2 and OCT4 are observed in numerous NCSC lines and commonly used as NCSC markers as well as pluripotency markers. It is known that migratory $\mathrm{NC}$ and postmigratory NC cells maintain a low SOX2 expression level (Nelms and Labosky, 2010). The expression level of these NCSC markers was high in induced cells by NC culture condition and reduced in NC-B culture condition (Fig.3, Table S3). On the other hand, NC-B culture condition promoted substantially increased activation of craniofacial NC gene subsets, including $D L X 1, D L X 2$, DLX3, DLX4, DLX5, DLX6, PITX1, PITX2, and HAND2, which are abundantly expressed in migratory and postmigratory NC cells in pharyngeal arches (Brunskill et al., 2014, Nelms and Labosky, 2010) (Fig.3, Table S3). Migratory NC cell markers, GATA4 and GATA6, were prominently expressed in the cells induced by NC-B culture condition (Fig.3, Table S3). A part of posterior HOX genes containing $H O X B 9$ and $H O X C 13$ were slightly activated; however, the expression levels of these genes were relatively lower than anterior $\mathrm{HOX}$ genes/cranial NC genes (Fig.3, Table S3).

These gene expression profiles indicate that the NC culture condition induced anterior premigratory NC cells and NCSCs and that NC-B culture condition induced pharyngeal arch NC cells and migratory NC cells. Thus, late exposure to BMP4 may have an important role in the transcriptional regulation of pharyngeal mesenchymal NC development.

\section{Differentiation of the induced NC cells into osteocytes and chondrocytes in vitro}

Cranial NC cells give rise to the majority of the craniofacial bone and cartilage (Santagati and Rijli, 2003). Therefore, we further evaluated the osteogenic and chondrogenic potential of NC cells induced by NC-B culture condition by further culturing for 21 days in an osteogenic or chondrogenic medium, respectively. Cells cultured in the osteogenic medium were stained deeply by alizarin red (Fig.4 A). Those cultured in the chondrogenic medium were stained deeply by both alcian blue (Fig.4 B) and safranine O (Fig.4 C). These results demonstrate that NC cells induced by NC-B culture condition are capable of differentiating into osteocytes and chondrocytes, indi- cating the NC-B culture condition induced a cranial mesenchyme population. It had been demonstrated that NCSCs induced without BMP4 were differentiated into mesenchymal stem cells by culturing in the medium containing $10 \%(\mathrm{v} / \mathrm{v}) \mathrm{FBS}$, and the mesenchymal stem cells were further differentiated over 3 or 4 weeks to osteocytes or chondrocytes by Dalton et al. (Menendez et al., 2013, Menendez et al., 2011). These results suggested that NC cells induced from human ESCs in NC and NC-B culture condition included anterior NCSCs and cranial mesenchyme in cranial regions.

\section{Discussion}

In the present study, we demonstrate that timely treatment with BMP4 after NC specification is able to induce a distinct subset of NC cells which express cranial positional information and pharyngeal mesenchymal genes in vitro. NC cells treated with BMP4 were capable of differentiating into both osteocytes and chondrocytes, indicating the successful induction of cranial NC. Thus, we describe the development of a differentiation protocol for cranial mesenchymal NC induction from hESCs, modifying the previous NCSC induction protocol using chemically-defined culture conditions.

Our differentiation strategy, late exposure to BMP4, coincides with the current model of intracellular and extracellular signaling during NC induction (Stuhlmiller and Garcia-Castro, 2012). Previous studies in vertebrates have identified numerous signaling factors, including FGF, Wnt, and BMP, involved in the specification and fate determination of NC cells in a different manner during gastrulation and neurulation. The canonical Wnt signaling is critical for the specification of NC during gastrulation (Garcia-Castro et al., 2002). Wnt signaling can inhibit BMP4 expression at early gastrula stages in Xenopus (Baker et al., 1999). Although there is no direct evidence that FGF signaling is involved in NC induction during mouse development, there is accumulating evidence for the role of FGF signaling during NC induction in Xenopus, chicken, and zebrafish (Stuhlmiller and Garcia-Castro, 2012). The activation of FGF signaling in the early embryo up-regulates both BMP and Wnt signaling (Garnett et al., 2012). We previously reported that Wnt signaling induces the development of anterior and posterior primitive streak cells from both mouse and hESCs under defined culture conditions (Nakanishi et al., 2009). Furthermore, BMP signaling is temporally activated during NC induction in Xenopus and chick embryos, with the inhibition of BMP signaling at the gastrula stage and activation of BMP and Wnt signaling at the neurula stage required (Patthey et al., 2009). These studies indicate that inhibition 
of BMP signaling is required for the specification of early NC and that BMP and Wnt signaling are required for the maintenance of NC population and further differentiation. We believe the following two-step NC induction method developed in the present study is compatible with these models: (1) activation of Wnt and FGF signaling from day 0 to day 8 for NC specification and (2) activation of BMP in combination with Wnt and FGF signaling from day 8 to day 12. Activating BMP4 signaling at specific time points appears critical for the induction of cranial mesenchyme from early NC cells.

Heterogeneity among $\mathrm{p} 75^{\mathrm{NTR}+}$ cells in induced NC populations has been reported in vitro, with both SOX10 expression and the ability to differentiate into NC derivatives (Curchoe et al., 2010). The expression of $P A X 3, S O X 9$, and $S O X 10$ in premigratory NC cells and that of $P A X 7, S O X 9, A P 2 \alpha$, and $p 75^{N T R}$ in migratory NC cells has been demonstrated in addition to the expression of $S O X 10$ in human embryos (Betters etal., 2010). Overall, these results indicate that SOX10 and other NC marker genes, in addition to $p 75^{N T R}$ and $H N K 1$, are required for the definitive characterization of NC cells in vivo and in vitro. Moreover, recent studies have reported the in vivo NC expression profiles of transcription factors and other related genes at both the mRNA and protein level in mouse and human embryos (Betters et al., 2010, Brunskill et al., 2014). Gene expression profiles in induced cells in vitro should be comprehensively analyzed and compared with recent in vivo findings. In the present study, the cranial mesenchyme NC population induced by BMP4 in the NC induction culture condition demonstrated a lower proportion of p $75^{\mathrm{NTR}+}$ cells compared with those induced without BMP4 in the NC induction culture condition. Induced cranial mesenchyme NC cells had substantial up-regulation of genes related to NC maintenance, migration, craniofacial positioning, and tooth development, whereas small changes in genes expressed in posterior NC positions, such as the vagal, trunk, or sacral NC, were observed. To the best of our knowledge, we were first to demonstrate high levels of MSX and DLX expression in NC cells directly induced from hESCs. The expression of anterior HOX genes is affected by retinoic acid treatment during NC induction (Fukuta et al., 2014). These findings indicate the potential utility of modulating signaling factors step-by-step in controlling regional characteristics of NC cells during directed induction in vitro.

In order to efficiently induce specific NC populations, such as cranial NC cells in vitro, the sequential gene expression changes occurring in vivo during embryonic development are required to be accurately replicated. Temporal prescription of extra cellular matrixes (ECM), growth factors and/or small molecules, which act as inhibitors or activators of signaling cascades, can control gene expression during directed differentiation. We previously demonstrated the induction of jaw cartilage with the expression of maxillofacial regional marker genes from Xenopus undifferentiated presumptive ectoderm in vitro using Activin $A$ and a sandwiched explant culture system (Furue et al., 2002). Furthermore, we also reported the induction of tooth development from mixed cultures of Activin A-treated dissociated cells and untreated cells of Xenopus undifferentiated presumptive ectoderm (Myoishi et al., 2004). In the present study, we induced cranial NC, particularly craniofacial mesenchyme, from hESCs. The combination of the methodology used in these culture systems and recent developments in the understanding of gene expression profiles in the early human embryo will facilitate the development of novel directed differentiation methods and promote an increased understanding of the mechanisms underlying the regulation of cranial NC development in humans. Although various methods for efficient directed differentiation of human pluripotent stem cells have been reported, numerous limitations remain. Almost all induction methods reported till date do not allow the analysis of the effects of ECM, growth factors, or small molecules because currently-used methods utilize undefined components, such as Matrigel and conditioned medium, or BSA at high concentrations. In order to analyze extracellular and intracellular signaling during differentiation more accurately, the use of completely chemically-defined culture conditions is preferable. Accordingly, we are currently developing a new defined culture method to control the induction of NC cells with the expression of positional information genes.

\section{Materials and Methods}

\section{Cell culture}

The human ES cell line, H9 (WA09, WISC Bank, WiCell Research Institute, Madison, WI, USA) was routinely maintained on mouse embryo fibroblast feeder cells inactivated by $\gamma$-irradiation (MEF CF-1, Applied StemCell, Inc., CA, USA) in a hESC expansion medium (KSR-based medium) consisting of DMEM/F12 medium (Life Technologies, CA, USA) supplemented with $20 \%$ KSR (Life Technologies Ltd, Paisley, UK), 0.1 mM 2-mercaptoethanol (Sigma, St. Louis, MO, USA), 2 mM L-glutamine (Gibco, NY, USA), $0.1 \mathrm{mM}$ non-essential amino acids (Gibco), and $5 \mathrm{ng} /$ $\mathrm{ml}$ human recombinant FGF-2 (Katayama Kagaku Kogyo, Japan) as previously described (Kinehara et al., 2013). The cells were split at a ratio of 1:8-1:10 every 6-7 days. Culture medium was replaced with hESF9, a growth-factor defined serum-free ESC culture medium, which we previously developed (Furue et al., 2008) at least $24 \mathrm{~h}$ before re-seeding for differentiation. The hESF9 medium consisted of ESF basal medium (Wako Pure Chemicals, Japan) supplemented with five factors $(10 \mu \mathrm{g} / \mathrm{ml}$ of human recombinant insulin, $5 \mu \mathrm{g} / \mathrm{ml}$ of human transferrin, $10 \mu \mathrm{M}$ of 2-mercaptoethanol, $10 \mu \mathrm{M}$ of 2-ethanolamine, $20 \mathrm{nM}$ of sodium selenite), $9.4 \mu \mathrm{g} / \mathrm{ml}$ of oleic acid conjugated with fatty acid-free bovine serum albumin (BSA), $100 \mathrm{ng} / \mathrm{ml}$ of bovine heparan sulfate sodium salt (all from Sigma), $0.1 \mathrm{mg} /$ $\mathrm{ml}$ of L-ascorbic acid 2-phosphate, and $10 \mathrm{ng} / \mathrm{ml}$ of human recombinant FGF-2. Cells were checked for mycoplasma infection every month using a MycoAlert Mycoplasma Detection Kit (Lonza). Identification of cells used in the present study confirmed the $\mathrm{H} 9$ cell line against the JCRB Cell Bank (National Institutes of Biomedical Innovation, Health and Nutrition, Japan). Human ESCs were used following the Guidelines for the utilization of hESCs of the Ministry of Education, Culture, Sports, Science and Technology of Japan after approval by the institutional ethical review board at National Institutes of Biomedical Innovation, Health and Nutrition.

\section{Differentiation of hESCs into NC cells in monolayer culture}

H9 cells were cultured under the modified protocol reported by Menendez et al. (Menendez et al., 2013, Menendez et al., 2011) for the differentiation of hESCs into NC cells. $\mathrm{H} 9$ cells at a cell density of $1 \times 10^{4}$ cells were dissociated into single cells by Accutase (EMD Millipore) and seeded on Matrigel (BD)-coated wells of 6-well plates in hESF9 medium and cultured for 1-2 days. Cells were then cultured in NC differentiation medium. NC differentiation medium consisted of DMEM/F12 (Cat. No. 10505 , supplemented with GlutaMAX-I, Invitrogen) containing $2 \%$ BSA (Cat. No. 82-067-3, EMD Millipore), 1× MEM non-essential amino acids (Invitrogen), $1 \times$ trace element A (Cellgro), $1 \times$ trace element B (Cellgro), $1 \times$ trace element $C$ (Cellgro), $0.1 \mathrm{mM}$ 2-mercaptoethanol, $10 \mu \mathrm{g} / \mathrm{ml}$ apotransferrin (Sigma), $50 \mu \mathrm{g} / \mathrm{ml}$ (+)-sodium L-ascorbate (Sigma), $10 \mathrm{ng} / \mathrm{ml}$ NRG1/HRG1 (R\&D), 200 ng/ml LONGR ${ }^{3}$ IGF-1 (Sigma), 8 ng/ml FGF-2, $25 \mathrm{ng} / \mathrm{ml}$ Wnt-3a (R\&D), and $20 \mu \mathrm{M}$ SB431542 (Tocris). Culture medium was replaced every day during differentiation. From day 8 to day 12, cells were cultured in NC differentiation medium supplemented with $100 \mathrm{ng} / \mathrm{ml}$ of BMP4 (NC-B condition) or without BMP4 (NC condition). 


\section{Differentiation of hESC-derived NC cells into osteocytes and chondrocytes}

Osteogenic or chondrogenic differentiation protocols were performed using osteogenic induction medium (StemPro Osteogenesis Differentiation kit, Life technologies) or chondrogenic induction medium (StemPro Chondrogenesis Differentiation kit, Life technologies), respectively. Alizarin Red (Wako) was used to detect osteocytes. Safranine O (Wako) and Alcian Blue solution (Sigma) were used to detect chondrocytes. Images of stained cells were obtained using a stereoscopic microscope (MZ12, Leica).

\section{Immunocytochemistry and flow cytometry}

Immunocytochemistry and flow cytometry were performed as previously described (Menendez et al., 2013). Monoclonal anti-HNK-1/N-CAM antibody (mouse IgM clone VC1.1, C6680, Sigma) and monoclonal anti-p75/NGFR antibody (mouse lgG clone ME20.4, AB-N07, Advanced Targeting Systems, San Diego, CA, USA) were used as primary antibodies. Alexa Fluor 488 labeled goat anti-mouse lgM antibody and Alexa Fluor 647 labeled goat anti-mouse IgG antibody were used for fluorescent detection. Nuclei were detected by Hoechst33342 staining. Images were obtained by fluorescent microscopy (Ti, Nikon). Flow cytometry data were acquired using a guava easyCyte $^{\mathrm{TM}} 8 \mathrm{HT}$ Flow Cytometer (EMD Millipore).

\section{Gene expression analysis}

Total RNA was extracted from undifferentiated hESCs or hESC-derived NC cells using AllPrep DNA/RNAMini kits (QIAGEN), treated with RNase-free DNasel (Invitrogen), and reverse transcribed with SuperScript VILO cDNA synthesis Kits (Invitrogen), according to the manufacturer's instructions. Synthesized cDNA was amplified using $\mathrm{RT}^{2}$ Profiler $^{\mathrm{TM}}$ PCR Arrays (Human Homeobox (HOX) Genes and custom designed array, QIAGEN) or genespecific primers (Invitrogen). The primers used in the present study are shown in Supplementary Table S1. Genes analyzed in the array are listed in Supplementary Table S2. Array data were analyzed using web-based software for cataloged and custom arrays ( $\mathrm{RT}^{2}$ Profiler ${ }^{\mathrm{TM}}$ PCR Array Data Analysis Ver. 3.5, QIAGEN).

\section{Author Contributions}

M.K.F. and H.N. conceived and supervised this study. S.M., M.S., K.O. and M.K. performed all cell culture experiments and related analyses. M.S. and M.K.F. supervised and performed the microarray analyses. All authors contributed to manuscript preparation.

\section{Acknowledgments}

We thank Jim Hackland, Christian Unger, and Peter W. Andrews for helpfuldiscussion. We thank HarukaAsano, Mari Wakabayashi, and Naoko Ueda for excellent technical support and Takayuki Fukuda for excellent support and discussion. The authors would like to thank Enago (www.enago. jp) for the English language review. This study was supported by grantsin-aid from the Ministry of Health, Labor and Welfare of Japan to M.K.F. and the Ministry of Education, Culture, Sports, Science and Technology of Japan to M.K.F. and M.S.

\section{References}

AIHARA, Y., HAYASHI, Y., HIRATA, M., ARIKI, N., SHIBATA, S., NAGOSHI, N., NAKANISHI, M., OHNUMA, K., WARASHINA, M., MICHIUE, T. et al. (2010). Induction of neural crest cells from mouse embryonic stem cells in a serum-free monolayer culture. Int J Dev Biol 54: 1287-1294.

AMIT, M., CARPENTER, M.K., INOKUMA, M.S., CHIU, C.-P., HARRIS, C.P., WAKNITZ, M.A., ITSKOVITZ-ELDOR, J. and THOMSON, J.A. (2000). Clonally Derived Human Embryonic Stem Cell Lines Maintain Pluripotency and Proliferative Potential for Prolonged Periods of Culture. Dev Biol 227: 271-278.

BAKER, J.C., BEDDINGTON, R.S.P. and HARLAND, R.M. (1999). Wnt signaling in Xenopus embryos inhibits Bmp4 expression and activates neural development. Genes Dev. 13: 3149-3159.

BETTERS, E., LIU, Y., KJAELDGAARD, A., SUNDSTR M, E. and GARC A-CASTRO,
M.I. (2010). Analysis of early human neural crest development. Dev Biol 344: 578-592.

BRUNSKILL, E.W., POTTER, A.S., DISTASIO, A., DEXHEIMER, P., PLASSARD, A., ARONOW, B.J. and POTTER, S.S. (2014). A gene expression atlas of early craniofacial development. Dev Biol 391: 133-146.

CURCHOE, C.L., MAURER, J., MCKEOWN, S.J., CATTAROSSI, G., CIMADAMORE, F., NILBRATT, M., SNYDER, E.Y., BRONNER-FRASER, M. and TERSKIKH, A.V. (2010). Early Acquisition of Neural Crest Competence During hESCs Neuralization. PLOS ONE 5: e13890.

FUKUTA, M., NAKAI, Y., KIRINO, K., NAKAGAWA, M., SEKIGUCHI, K., NAGATA, S., MATSUMOTO, Y., YAMAMOTO, T., UMEDA, K., HEIKE, T. et al. (2014). Derivation of Mesenchymal Stromal Cells from Pluripotent Stem Cells through a Neural Crest Lineage using Small Molecule Compounds with Defined Media. PLOS ONE 9: e112291.

FURUE, M. (2012). Monolayer Culture Condition for Mouse Embryonic Stem Cells Differentiation into Neural Crest Cells (Method). In Stem Cells and Cancer Stem Cells, Volume 5, vol. 5 (ed. HAYAT, M. A.). Springer Netherlands, pp.233-240.

FURUE, M. and ASASHIMA, M. (2004). 46 - Isolation of Pluripotential Stem Cells from Xenopus Embryos. In Handbook of Stem Cells, (ed. LANZA, R.GEARHART, J.HOGAN, B.MELTON, D.PEDERSEN, R.THOMSON, J. and WEST, M.). Academic Press, Burlington, pp.483-492.

FURUE, M., MYOISHI, Y., FUKUI, Y., ARIIZUMI, T., OKAMOTO, T. and ASASHIMA, M. (2002). Activin A induces craniofacial cartilage from undifferentiated Xenopus ectoderm in vitro. Proc Natl Acad Sci USA 99: 15474-15479.

FURUE, M.K., NA, J., JACKSON, J.P., OKAMOTO, T., JONES, M., BAKER, D., HATA, R., MOORE, H.D., SATO, J.D. and ANDREWS, P.W. (2008). Heparin promotes the growth of human embryonic stem cells in a defined serum-free medium. Proc Natl Acad Sci USA 105: 13409-13414.

GAJAVELLI, S., WOOD, P.M., PENNICA, D., WHITTEMORE, S.R. and TSOULFAS, P. (2004). BMP signaling initiates a neural crest differentiation program in embryonic rat CNS stem cells. Exp Neurol 188: 205-223.

GARCIA-CASTRO, M.I., MARCELLE, C. and BRONNER-FRASER, M. (2002). Ectodermal Wnt function as a neural crest inducer. Science 297: 848-851.

GARNETT, A.T., SQUARE, T.A. and MEDEIROS, D.M. (2012). BMP, Wnt and FGF signals are integrated through evolutionarily conserved enhancers to achieve robust expression of Pax3 and Zic genes at the zebrafish neural plate border. Development 139: 4220-4231.

ISHII, M., HAN, J., YEN, H.Y., SUCOV, H.M., CHAI, Y. and MAXSON, R.E., JR. (2005). Combined deficiencies of Msx1 and Msx2 cause impaired patterning and survival of the cranial neural crest. Development 132: 4937-4950.

KAMIYA, D., BANNO, S., SASAI, N., OHGUSHI, M., INOMATA, H., WATANABE, K., KAWADA, M., YAKURA, R., KIYONARI, H., NAKAO, K. et al. (2011). Intrinsic transition of embryonic stem-cell differentiation into neural progenitors. Nature 470: 503-509.

KINEHARA, M., KAWAMURA, S., TATEYAMA, D., SUGA, M., MATSUMURA, H., MIMURA, S., HIRAYAMA, N., HIRATA, M., UCHIO-YAMADA, K., KOHARA, A. et al. (2013). Protein kinase $C$ regulates human pluripotent stem cell self-renewal. PLOS ONE 8: e54122.

LEE, G., CHAMBERS, S.M., TOMISHIMA, M.J. and STUDER, L. (2010). Derivation of neural crest cells from human pluripotent stem cells. Nat Protoc 5: 688-701.

MENENDEZ, L., KULIK, M.J., PAGE, A.T., PARK, S.S., LAUDERDALE, J.D., CUNNINGHAM, M.L. and DALTON, S. (2013). Directed differentiation of human pluripotent cells to neural crest stem cells. Nat Protoc 8: 203-212.

MENENDEZ, L., YATSKIEVYCH, T.A., ANTIN, P.B. and DALTON, S. (2011). Wnt signaling and a Smad pathway blockade direct the differentiation of human pluripotent stem cells to multipotent neural crest cells. Proc Natl Acad Sci USA 108: 19240-19245.

MORIKAWA, S., MABUCHI, Y., NIIBE, K., SUZUKI, S., NAGOSHI, N., SUNABORI, T., SHIMMURA, S., NAGAI, Y., NAKAGAWA, T., OKANO, H. et al. (2009). Development of mesenchymal stem cells partially originate from the neural crest. Biochem Biophys Res Commun 379: 1114-1119.

MYOISHI, Y., FURUE, M., FUKUI, Y., OKAMOTO, T. and ASASHIMA, M. (2004). Induction of tooth and eye by transplantation of activin A-treated, undifferentiated presumptive ectodermal Xenopus cells into the abdomen. Int $J$ Dev Biol 48: 1105-1112.

NAGASE, T., SANAI, Y., NAKAMURA, S., ASATO, H., HARII, K. and OSUMI, N. (2003). 


\section{S. Mimura et al.}

Roles of HNK-1 carbohydrate epitope and its synthetic glucuronyltransferase genes on migration of rat neural crest cells. J. Anatomy 203: 77-88.

NAKANISHI, M., KURISAKI, A., HAYASHI, Y., WARASHINA, M., ISHIURA, S., KUSUDA-FURUE, M. and ASASHIMA, M. (2009). Directed induction of anterior and posterior primitive streak by Wnt from embryonic stem cells cultured in a chemically defined serum-free medium. FASEB J 23: 114-122.

NELMS, B.L. and LABOSKY, P.A. (2010). Transcriptional Control of Neural Crest Development. 2010 by Morgan \& Claypool Life Sciences., San Rafael CA.

PARK, B.K., SPERBER, S.M., CHOUDHURY, A., GHANEM, N., HATCH, G.T., SHARPE, P.T., THOMAS, B.L. and EKKER, M. (2004). Intergenic enhancers with distinct activities regulate Dlx gene expression in the mesenchyme of the branchial arches. Dev Biol 268: 532-545.

PATTHEY, C., EDLUND, T. and GUNHAGA, L. (2009). Wnt-regulated temporal control of BMP exposure directs the choice between neural plate border and epidermal fate. Development 136: 73-83.

PICCOLO, S., SASAI, Y., LU, B. and DE ROBERTIS, E.M. (1996). Dorsoventral patterning in Xenopus: inhibition of ventral signals by direct binding of chordin to BMP-4. Cell 86: 589-598.

SANTAGATI, F. and RIJLI, F.M. (2003). Cranial neural crest and the building of the vertebrate head. Nat Rev Neurosci 4: 806-818.

STUHLMILLER, T.J. and GARCIA-CASTRO, M.I. (2012). Current perspectives of the signaling pathways directing neural crestinduction. Cell Mol Life Sci69:3715-3737.

ZIMMERMAN, L.B., DE JESUS-ESCOBAR, J.M. and HARLAND, R.M. (1996). The Spemann organizer signal noggin binds and inactivates bone morphogenetic protein 4. Cell 86: 599-606. 


\section{Further Related Reading, published previously in the Int. J. Dev. Biol.}

Generation of pluripotent stem cells via protein transduction

Xia Li, Pengfei Zhang, Chao Wei and Yunhai Zhang

Int. J. Dev. Biol. (2014) 58: 21-27

http://www.intjdevbiol.com/web/paper/140007XL

\section{BMP4 regulation of human trophoblast development}

Yingchun Li and Mana M. Parast

Int. J. Dev. Biol. (2014) 58: 239-246

http://www.intjdevbiol.com/web/paper/130341mp

Feeder- and serum-free establishment and expansion of human induced pluripotent stem cells

Mehdi Totonchi, Adeleh Taei, Ali Seifinejad, Mohammadsharif Tabebordbar, Hassan Rassouli, Ali Farrokhi, Hamid Gourabi, Nasser Aghdami, Ghasem Hosseini-Salekdeh and Hossein Baharvand

Int. J. Dev. Biol. (2010) 54: 877-886

doi: $10.1387 / \mathrm{ijdb} .092903 \mathrm{mt}$

Matrigel supports neural, melanocytic and chondrogenic differentiation of trunk neural crest cells

Ana B. Ramos-Hryb, Meline C. Da-Costa, Andréa G. Trentin and Giordano W. Calloni

Int. J. Dev. Biol. (2013) 57: 885-890

doi: $10.1387 / \mathrm{ijdb} .130206 \mathrm{gw}$

Long-term serial cultivation of mouse induced pluripotent stem cells in serum-free and feeder-free defined medium Sachiko Yamasaki, Kou Nabeshima, Yusuke Sotomaru, Yuki Taguchi, Hanae Mukasa, Miho K. Furue, J. Denry Sato and Tetsuji Okamoto Int. J. Dev. Biol. (2013) 57: 715-724

http://www.intjdevbiol.com/web/paper/130173to

Induction of intermediate mesoderm by retinoic acid receptor signaling from differentiating mouse embryonic stem cells Shiho Oeda, Yohei Hayashi, Techuan Chan, Minoru Takasato, Yuko Aihara,Koji Okabayashi, Kiyoshi Ohnuma and Makoto Asashima Int. J. Dev. Biol. (2013) 57: 383-389

http://www.intjdevbiol.com/web/paper/130058ma

Signaling pathways during maintenance and definitive endoderm differentiation of embryonic stem cells Lina Sui, Luc Bouwens and Josué K. Mfopou

Int. J. Dev. Biol. (2013) 57: 1-12

http://www.intjdevbiol.com/web/paper/120115ls
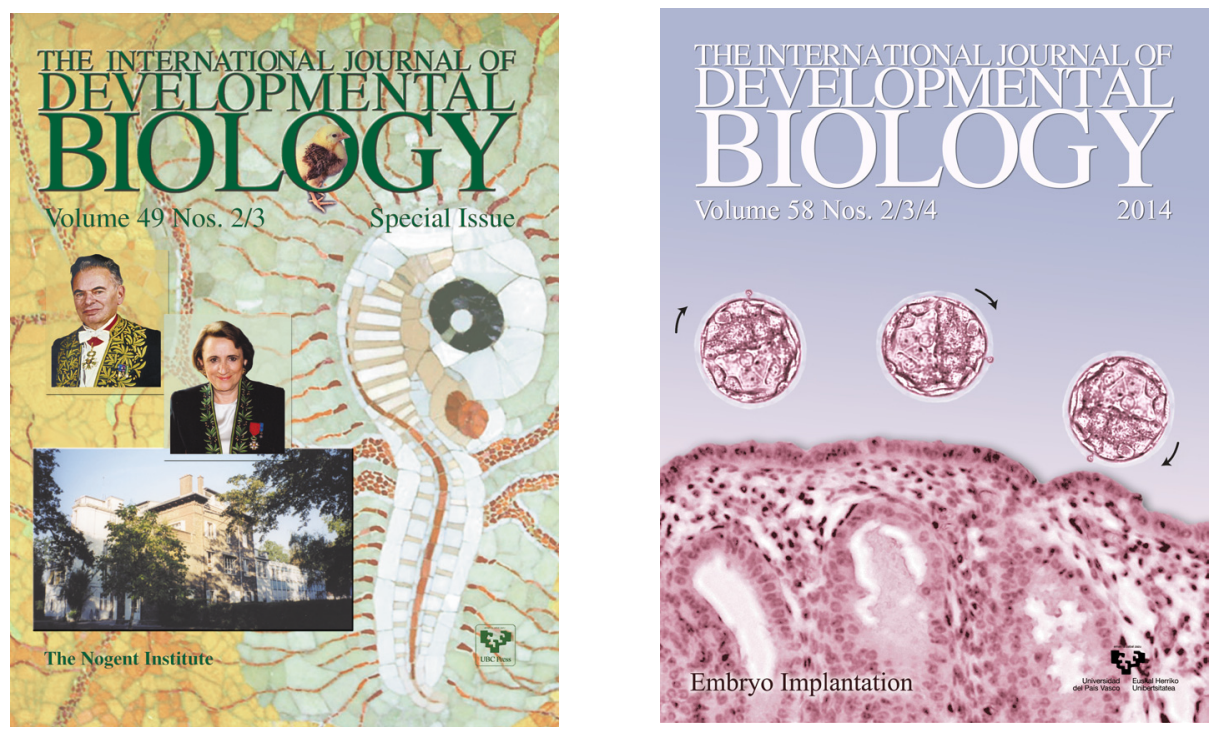